\title{
THE BIRTH PROCESS OF PERIODIC ORBITS IN NON-TWIST MAPS
}

\author{
J.P. VAN DER WEELE and T.P. VALKERING \\ Center for Theoretical Physics, University of Twente, P.O. Box 217, 7500 AE Enschede, \\ The Netherlands
}

Received 22 May 1990

We study the birth process of periodic orbits in non-twist systems, by means of a model map which contains all the typical features of such a system. The most common form of the birth process. or standard scenario, is described in detail. This scenario involves several steps: first one "dimerized" chain of saddle-center pairs is born, then a second, and eventually these two chains are reconnected into two Poincaré-Birkhoff chains.

We also discuss several variations on this standard scenario. These variations can give rise to arbitrarily many chains, intertwined in a complex fashion, and the reconnection of these chains can be highly non-trivial.

Finally we study the effect of dissipation on the birth process. For sufficiently small dissipation one can still recognize the birth and reconnection processes, but with several new features. In the first place, the chains do not consist anymore of conservative saddles and centers, but rather of dissipative saddles and nodes. Furthermore, the dissipation destroys the symmetry between the inner and outer chains, and as a result the reconnection does not take place in one single step anymore, but in three.

\section{Introduction}

This paper is about the birth of periodic orbits in non-twist maps. It is closely related to a previous paper [1] in which we demonstrated the birth process of twin Poincaré-Birkhoff chains in the area-preserving Hénon map, but while our primary concern there was the connection with the $1: 3$ resonance, we now want to explain the typical features of the birth itself. Moreover, we shall discuss the influence of dissipation upon the birth process. We begin by giving an outline of the problem, including a short review of our earlier results.

Consider an elliptic fixed point of an integrable area-preserving map. The orbits in the neighbourhood of this point circle around it, and their motion can be modelled by

$$
\left\{\begin{array}{l}
r^{\prime}=r, \\
\varphi^{\prime}=\varphi+2 \pi R\left(r^{\prime}\right) .
\end{array}\right.
$$


That is, the radius $r$ is kept constant and the angle $\varphi$ is increased by an amount $2 \pi R\left(r^{\prime}\right)$ at each step. The value of $R$ (or rather its reciprocal value) tells us how may steps it takes to make one full revolution around the origin, and in general this number will vary from orbit to orbit. We distinguish between two cases: the monotonic case, in which $R(r)$ is a monotonically increasing or decreasing function of $r$, and the non-monotonic case, in which $R(r)$ has a minimum or a maximum at a certain non-zero value $r=r_{0}$. In the monotonic case the map (1) is called a twist map, and in the non-monotonic case it is called a non-twist map, i.e. we have a non-twist situation at $r=r_{0}$.

Let us now add a perturbation to our map,

$$
\left\{\begin{array}{l}
r^{\prime}=r+\varepsilon f_{1}(r, \varphi), \\
\varphi^{\prime}=\varphi+2 \pi R\left(r^{\prime}\right)+\varepsilon f_{2}\left(r^{\prime}, \varphi\right),
\end{array}\right.
$$

in such a way that the integrability is broken but the area-preserving property remains intact. It is understood that $f_{1}$ and $f_{2}$ are periodic functions in $\varphi$ with period $2 \pi$, and that $f_{1}(0, \varphi)=f_{2}(0, \varphi)=0$ so that the origin at $r=0$ is still a fixed point.

In the monotonic case the famous Poincaré-Birkhoff theorem [2] then states that the orbits with a rational value of $R$ break up into chains of centers (or "islands") and saddles, the so-called Poincaré-Birkhoff chains, or PB chains for short. The equally famous KAM theorem [2] asserts that in between these PB chains for sufficiently small $\varepsilon$ we still have unbroken (just slighly deformed) orbits with irrational winding number $\rho$. The winding number $\rho$ of an orbit is defined as

$$
\rho=\lim _{n \rightarrow \infty} \frac{\varphi_{n}-\varphi_{0}}{2 \pi n},
$$

and can easily be determined numerically by choosing a starting point on the orbit (e.g. the point where the orbit intersects the $x$-axis) and then applying the map ad infinitum. For $\varepsilon=0$ the above definition of $\rho$ coincides with $R(r)$. For non-zero $\varepsilon$ the plot of $\rho$ against $r$ will deviate from $R(r)$, forming horizontal plateaus at the rational values, whenever we cut through an island of a Poincaré-Birkhoff chain. This is illustrated in fig. 1, where we show the neighbourhood of the origin for a typical twist map.

Let us next consider the non-monotonic case. Adding a perturbation to the map still yields Poincaré-Birkhoff chains at the slopes of $R(r)$, where (locally) the twist condition is satisfied just as in the previous case. However, the Poincaré-Birkhoff theorem does not tell us anything about the non-twist situation at the top of $R(r)$. In fig. 2 we show the neighbourhood of the origin for a typical non-twist map. Between the two PB chains of period 10 lies a 

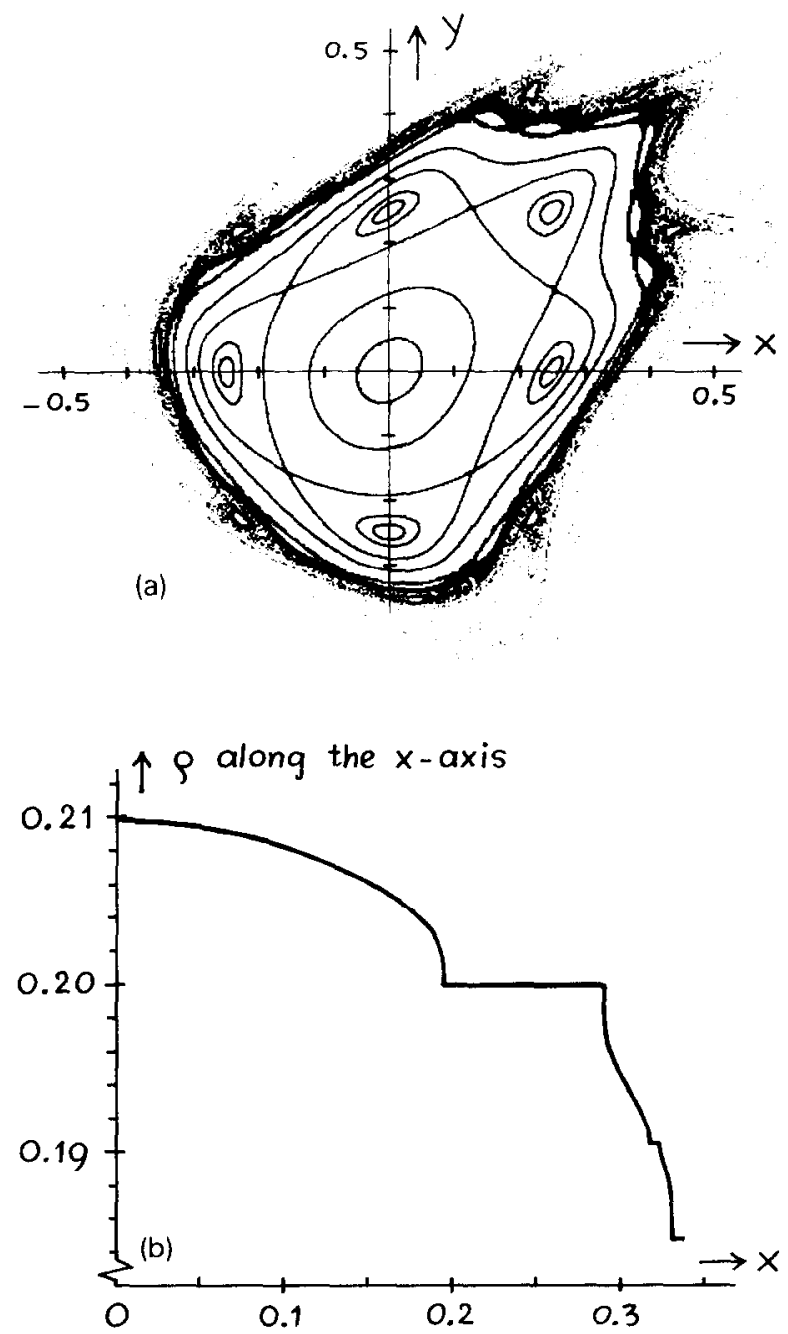

Fig. 1. (a) The neighbourhood of the origin for a typical twist map, the Henon map (5) at $C=0.25$. One clearly sees a Poincaré-Birkhoff chain of period 5 . In the dark zone which marks the boundary of the stable region around the origin one can also see a PB chain of period 16, and (less clearly) the remnants of a PB chain of period 11.

(b) The corresponding plot of the rotation number $\rho$ along the $x$-axis. The plateaus at $\rho=\frac{1}{5}$, $\rho=\frac{4}{21}=0.1905$ and $\rho=\frac{3}{16}=0.1875$ correspond to islands of PB chains.

non-twist region, which (in order not to give away our results too soon) we have left blank.

So what happens at the top? This is the central question of this paper. In section 2 , to get a first idea what the answer looks like, we discuss two examples of non-twist maps. Then, in section 3, we construct a model which exposes the anatomy of the problem and makes it possible to handle it 

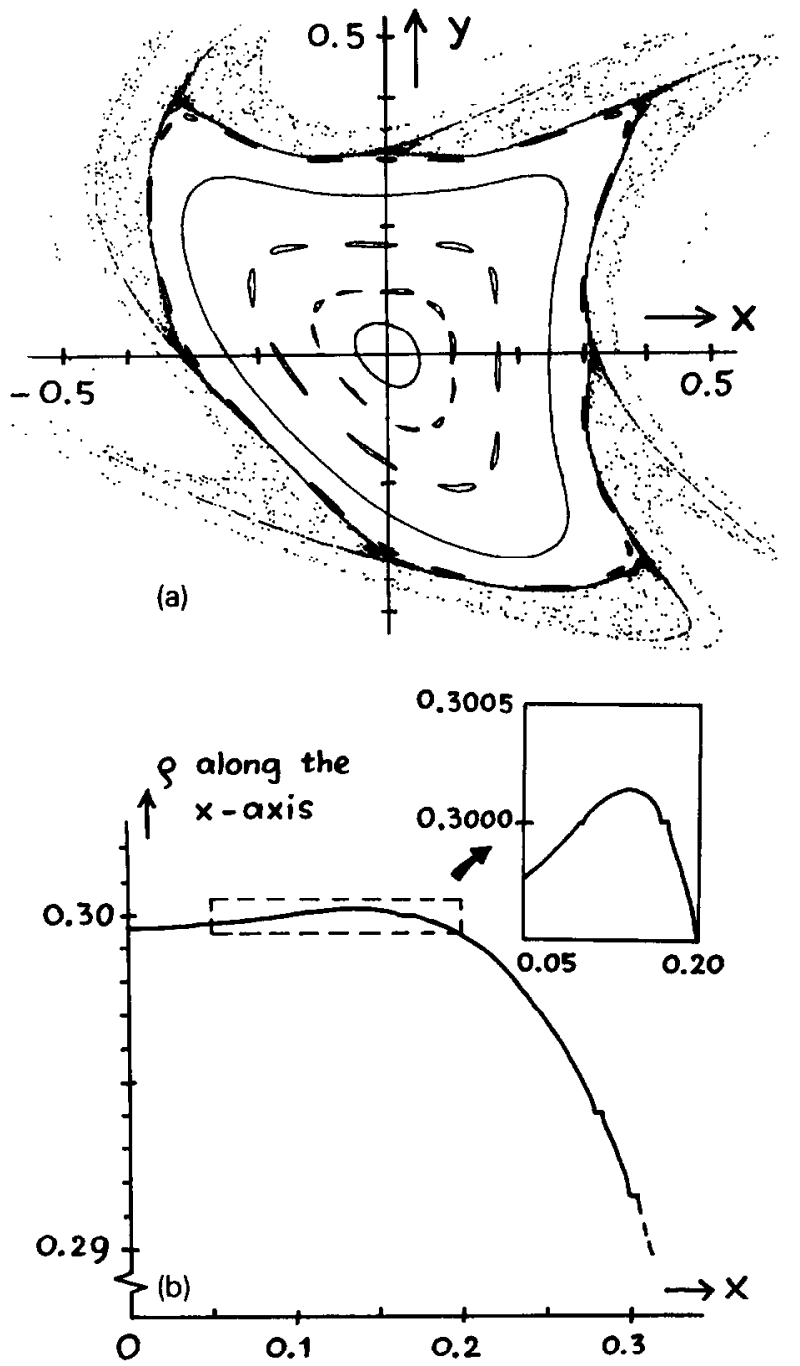

Fig. 2. (a) The neighbourhood of the origin for a typical non-twist map, the Hénon map (5) at $C=-0.307$. Note that inside the stable island we have two Poincaré-Birkhoff chains of period 10 , represented by the two rows of 10 islands each (the saddles and saddle connections have not been drawn). Near the boundary one can also see a PB chain of period 24.

(b) The corresponding plot of the rotation number along the $x$-axis, with a blow-up of the top. The plateaus at $\rho=\frac{3}{10}$ mark the islands of the two PB chains. Towards the right one can also discern plateaus at $\rho=\frac{5}{17}=0.2941$ and $\rho=\frac{7}{24}=0.2917$.

analytically. In section 4 we calculate the bifurcation diagram for this model, and in section 5 we comment upon its generality. In section 6 we discuss the influence of dissipation. Finally, in section 7 we draw some general conclusions. 


\section{Two examples}

In this section we will briefly review two examples of non-twist maps, in order to get an idea what happens at the maximum of $R(r)$.

The first example comes from the work of Howard and Hohs [3], who considered the map

$$
\left\{\begin{array}{l}
r^{t}=r-\varepsilon \sin \varphi \\
\varphi^{\prime}=\varphi+r^{\prime}\left(1-\alpha r^{\prime}\right),
\end{array}\right.
$$

where $\varepsilon$ and $\alpha$ are two positive parameters. (We have translated the map into our own notation for uniformity.) This is a non-twist map, since the function $R(r)=r(1-\alpha r) / 2 \pi$ has a maximum value $1 / 8 \pi \alpha$ at $r=1 / 2 \alpha$. The maximum goes upwards (and to larger values of $r$ ) for decreasing values of the parameter $\alpha$. So one may anticipate that new periodic orbits, with rotation number $p / q$, should be born when the maximum goes through a rational value $p / q$. Indeed, Howard and Hohs found that at $\alpha=(8 \pi p / q)^{-1}$ two dimerized chains, consisting of $q$ centers and $q$ saddles each, were born simultaneously. In this type of chain the centers and saddles come in pairs, or dimers. They also found that, upon further decreasing the value of $\alpha$, the saddles and centers reconnect to form two Poincaré-Birkhoff chains. This is (schematically) illustrated in fig. 3a. This figure also serves to show the difference between dimerized and PB chains, picturesquely (and very aptly) called "pendants" and "necklaces" in the language of ref. [4].

The second example comes from our own work on the $1: 3$ resonance $[1,5]$. In particular, we studied the Hénon map

$$
\left\{\begin{array}{l}
x^{\prime}=2 C x+2 x^{2}-y \\
y^{3}=x
\end{array}\right.
$$

which has a non-monotonic winding number $\rho(r)$ for the parameter interval $-\frac{1}{4}>C>-\frac{1}{2}$. This is illustrated in fig. $2 b$ for $C=-0.307$. The maximum of $\rho(r)$ moves upward (and to larger values of $r$ as well) for decreasing values of $C$, just as in the previous example. At $C \approx-0.3063$ the maximum goes through the rational value $\frac{3}{10}$, and in fig. $3 b$ we show schematically what happens. First one dimerized chain is born, then a second, and after that these two chains reconnect to form two $\mathrm{PB}$ chains. We will call this the standard scenario of the birth process. The corresponding real-life pictures, together with plots of the maximum of $\rho(r)$, are presented in fig. 4 where we see five stages of the birth process (the same five stages as in fig. 3b). Between figs. $4 a$ and $b$ the maximum of $\rho(r)$ reaches the value $\frac{3}{10}$ and the first dimerized chain is born, in fig. $4 \mathrm{c}$ the second one has been born, in fig. $4 \mathrm{~d}$ we are on the threshold of 

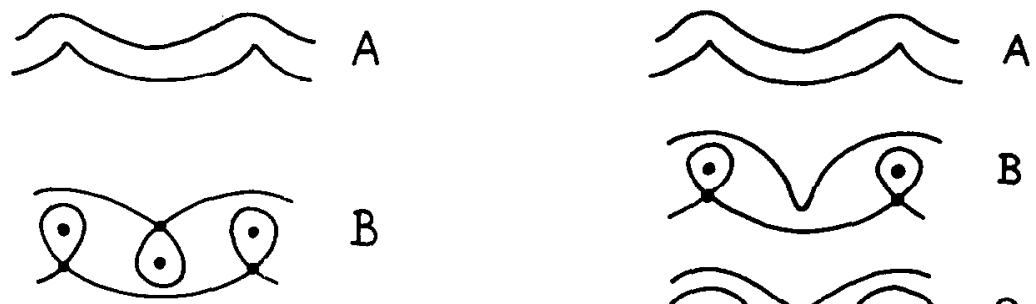

B

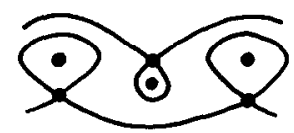

C
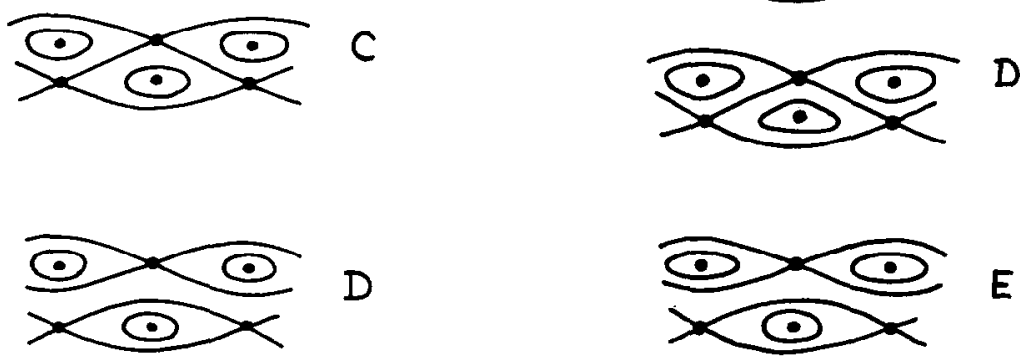

D

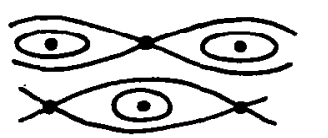

$E$

(a)

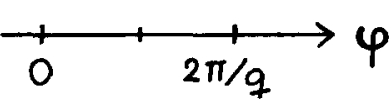

(b)

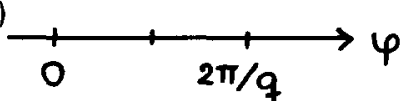

Fig. 3. (a) Four stages of the birth and reconnection process of periodic orbits in the non-twist map (4). Since the structures are periodic in $\varphi$ with period $2 \pi / q$, we have restricted ourselves to the fundamental interval $0 \leqslant \varphi<2 \pi / q$. We have: A. prelude, B. two dimerized chains, C. the reconnection threshold, D. two Poincaré-Birkhoff chains. This sequence is not the most generic one, since in general the two dimerized chains need not be born simultaneously.

(b) Five stages of the birth and reconnection process in map (5). Here the former stage B of (a) is split into two stages. We have: A. prelude, B. one dimerized chain, C. two dimerized chains, D. the reconnection threshold, E. two Poincaré-Birkhoff chains. These five pictures correspond to figs. 4a-e, respectively.

reconnection, and finally in fig. 4e we have two Poincaré-Birkhoff chains. This "movie" has been taken from ref. [1], and the interested reader will find a more detailed commentary there.

The main difference between the two examples above is that in the first example the dimerized chains are born simultaneously (fig. 3a), whereas in the second example they are born one after another (fig. $3 b$ ). The simultaneousness of the first example, which is obviously not generic, can be explained by the fact that the map (4) contains a superfluous symmetry. In order to get generic behaviour onc must choose a model which does not contain such a symmetry; map (5) of the second example apparently does the job. Getting rid of non-generic symmetries is an important part of the art of model building, and we shall return to it in section 6 ; in fact, we shall be compelled to return to it, being faced again with a nasty symmetry. 

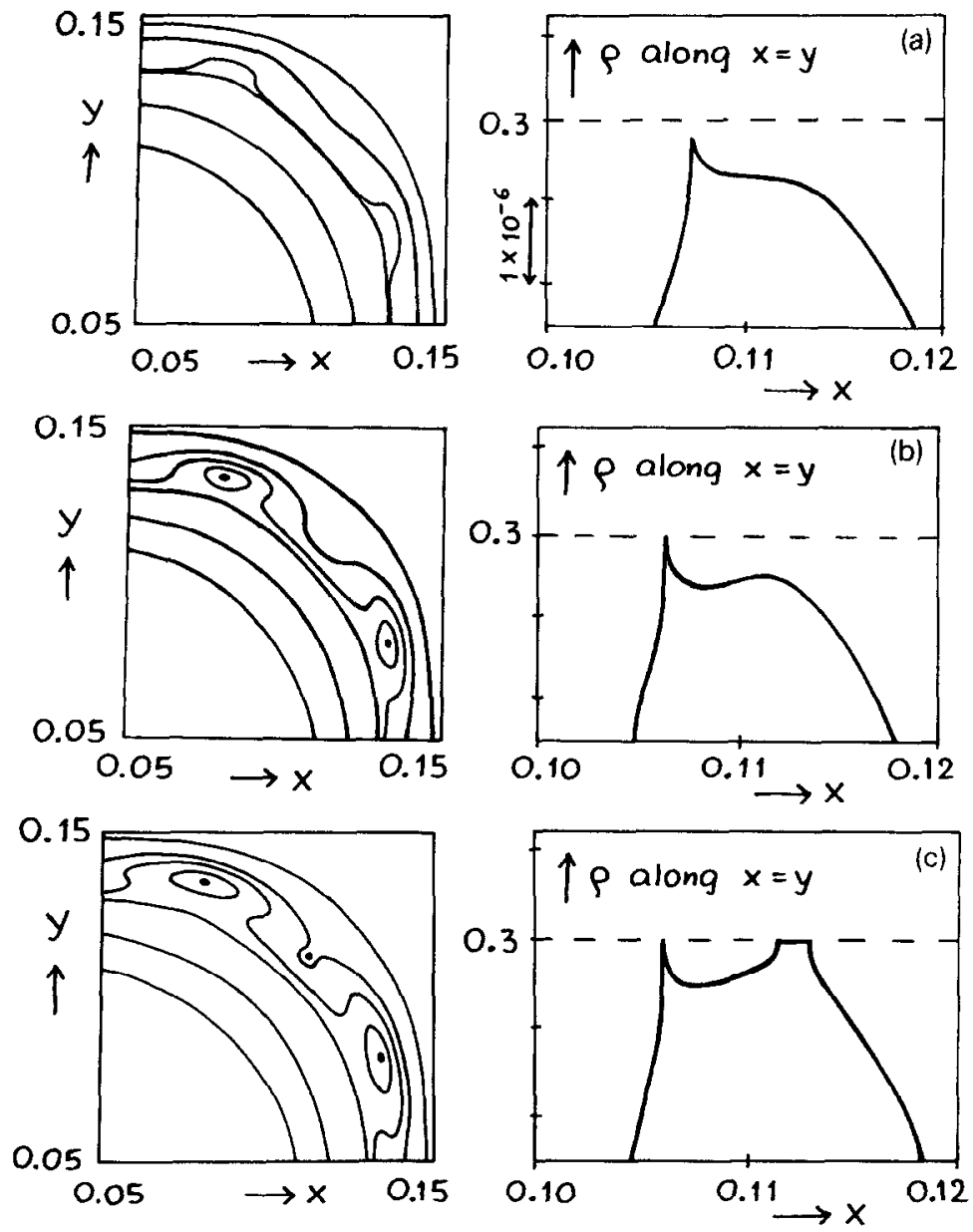

Fig. 4. (a) The Henon map (5) at $C=-0.306286$. In the left-hand picture we show a part of the $(x, y)$ plane, exhibiting orbits with blobs where the saddle-center bifurcations for the first dimerized chain are going to take place. The right-hand picture shows the rotation number $\rho$ along the line $x=y$.

(b) The same as in (a), but now at $C=-0.306288$. The first dimerized chain has been born. One heteroclinic saddle connection crosses the line $x=y$, yielding the sharp peak at $\rho=\frac{3}{i i}$ in the right-hand picture.

(c) The same as in (a) and (b), but now at $C=-0.306290$. The second dimerized chain has been born, which has a saddle-center pair on the line $x=y$. This yields a plateau in the plot of $\rho$ along this line.

(d) The same as in (a)-(c), but now at $C=-0.306297$. The two dimerized chains are on the verge of being reconnected into two Poincaré-Birklıoff chains. The plot of $\rho$ along the line $x=y$ shows an uninterrupted plateau.

(e) The same as in (a)-(d), but now at $C=-0.306299$. We have now two Poincaré-Birkhoff chains, separated hy an orbit with $\rho>\frac{3}{10}$. This is also clear from the plot of $\rho$ along $x=y$, where we see that the maximum has broken through the value $\frac{3}{10}$. The solid dot indicates that the line $x=y$ crosses a saddle of the outer PB chain, with negligible width. 

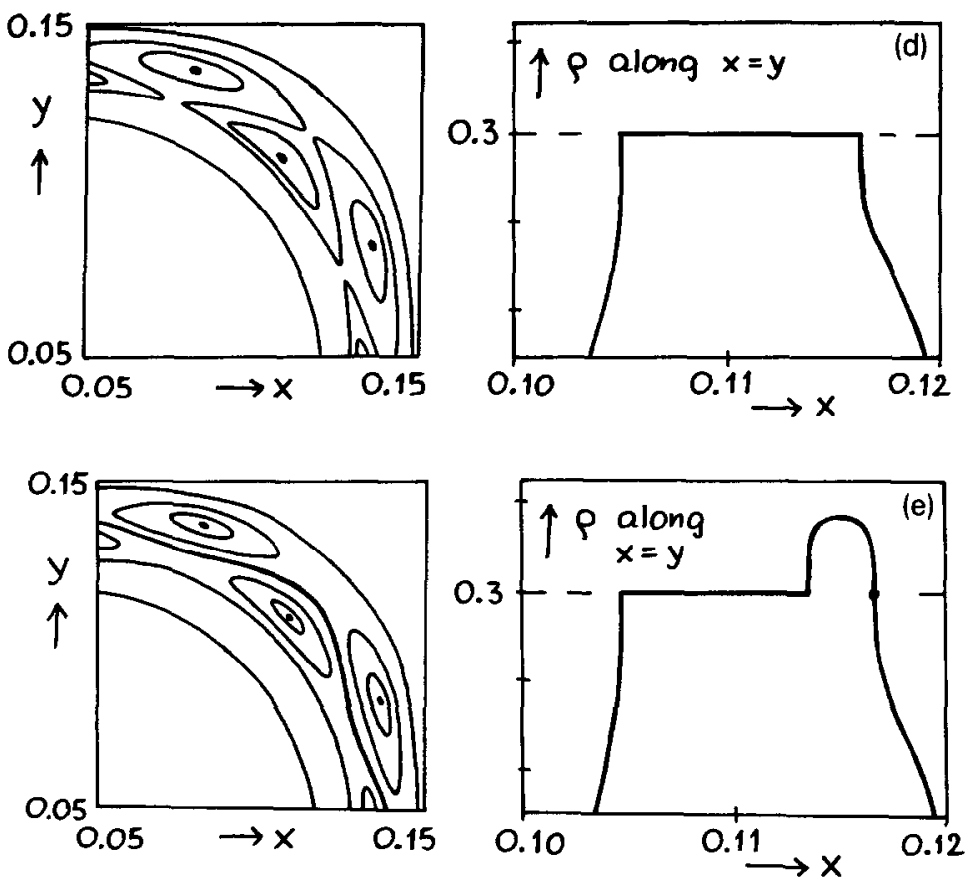

Fig. 4. (cont.).

\section{Constructing a typical model}

In this section we shall construct a model map which combines all the features of the previous two examples (in particular the second one) in such a way that we can easily handle them.

First of all, our model map should be a non-twist map, in which we can vary the height of the maximum of $R(r)$. We choose

$$
R(r)=\frac{p}{q}-f\left(r-r_{0}\right)+\frac{\mu}{2 \pi},
$$

where $f\left(r-r_{0}\right)$ is a non-negative function with $f(0)=0$, of the general form

$$
f\left(r-r_{0}\right)=\beta\left|r-r_{0}\right|^{2}, \quad z>0 .
$$

In this paper we will take $z=2$, so that $R(r)$ has a quadratic maximum at $r=r_{0}$. Its height is $p / q+\mu / 2 \pi$, which we can raise or lower with the parameter $\mu$. The value of $\beta$ is not very relevant; unless stated otherwise we will take it to be 0.2 . It may be noted that the maximum does not shift to the right or left, but always remains at the same value of $r$, in contrast to our 
examples in the previous section. This, however, proves to be not an essential restriction.

Having secured the non-twist character of our model we now want to add a perturbation of the form $\varepsilon f_{2}(r, \varphi)$ to make the mapping non-integrable. This perturbation must be periodic in $\varphi$, with zero average, and it should be compatible with a $p / q$ orbit. We will take it to be of the form

$$
f_{2}(r, \varphi)=F(r) g(\varphi)
$$

with $g(\varphi)$ a suitable periodic function, for instance $g(\varphi)=\cos (q \varphi)$. Inserting the above expressions in the general mapping (2) we find for the second line

$$
\varphi^{\prime}=\varphi+2 \pi\left(\frac{p}{q}-\beta\left(r^{\prime}-r_{0}\right)^{2}\right)+\mu+\varepsilon F\left(r^{\prime}\right) g(\varphi) .
$$

The first line then follows automatically from the requirement that the map should be area-preserving. This means [6] that the mapping must be governed by a generating function $S\left(r^{\prime}, \varphi\right)$ as follows:

$$
\left\{\begin{array}{l}
r=\frac{\partial S\left(r^{\prime}, \varphi\right)}{\partial \varphi} \\
\varphi^{\prime}=\frac{\partial S\left(r^{\prime}, \varphi\right)}{\partial r^{\prime}} .
\end{array}\right.
$$

Comparing (9) and (10b) we immediately see that $S\left(r^{\prime}, \varphi\right)$ must be of the form

$$
S\left(r^{\prime}, \varphi\right)=\varphi r^{\prime}+2 \pi\left(\frac{p}{q} r^{\prime}-\frac{\beta}{3}\left(r^{\prime}-r_{0}\right)^{3}\right)+\mu r^{\prime}+\varepsilon g(\varphi) \int_{0}^{r^{\prime}} F(x) \mathrm{d} x
$$

and hence, from (10a),

$$
r=r^{\prime}+\varepsilon g_{\varphi}(\varphi) \int_{0}^{r^{\prime}} F(x) \mathrm{d} x
$$

where the index $\varphi$ denotes differentiation with respect to $\varphi$. Of course, to get a regular (forward) mapping we want (12) to be invertible; for sufficiently small $\varepsilon$ this will not be a problem. Also, we require the integral in (12) to be zero at $r^{\prime}=0$, so that the origin remains a fixed point under the perturbation. A reasonable choice then is to take a member of the following family:

$$
F(x)=x^{k}, \quad k=0,1,2, \ldots
$$


For the time being we will take the simplest member of all: $k=0$, so $F\left(r^{\prime}\right)=1$ and $\int_{0}^{r^{\prime}} F(x) \mathrm{d} x=r^{\prime}$. Our model map then reads

$$
\left\{\begin{array}{l}
r^{\prime}=\frac{r}{1+\varepsilon g_{\varphi}(\varphi)}, \\
\varphi^{\prime}=\varphi+2 \pi\left(\frac{p}{q}-\beta\left(r^{\prime}-r_{0}\right)^{2}\right)+\mu+\varepsilon g(\varphi) .
\end{array}\right.
$$

In the next sections we will work out this model in detail, and we postpone a discussion about its merits or imperfections until we encounter them. For the moment we just want to draw attention to the fact that our model map has two relevant parameters: $\mu$ with which we can lower or raise the top of $R\left(r^{\prime}\right)$, and $\varepsilon$ which tells us how far we are from integrability. This gives us more freedom to play around than we had in the examples in the previous section, which contained only one free parameter. In other words, we have disentangled two separate effects which in the Hénon map were hidden in one single parameter. This is an important step forward.

\section{The bifurcation diagram}

In this section we shall calculate the bifurcation diagram in the $\varepsilon \mu$ plane. That is, we are going to determine the regions in the $\varepsilon \mu$ plane for which there are respectively zero, two and four $p / q$ orbits. From the examples in section 2 we already know that in the region of two orbits we will have one dimerized chain, and in the region of four orbits we will either have two dimerized chains or two PB chains.

For $\varepsilon=\mu=0$ any $p / q$ orbit (given that it exists) will lie on the circle $r=r_{0}$, and it will consist of $q$ points uniformly distributed on this circle, i.e. with angles $2 \pi p / q$ between them. For small non-zero $\varepsilon$ and $\mu$ the $p / q$ orbit will not be much different, and we may write it as follows:

$$
\begin{aligned}
& r_{i}=r_{0}+x+\rho_{i}, \\
& \varphi_{i}=\varphi_{0}+i 2 \pi p / q+\psi_{i},
\end{aligned}
$$

with $i=1,2, \ldots, q$. The deviations from the non-perturbed orbit have been separated in a uniform part (which does not depend on $i$ ) and a non-unifrom part (which does). The uniform part in the radial direction, denoted by $x$, is of the order $\varepsilon^{1 / 2}$ or $\mu^{1 / 2}$, or shortly $\mathcal{O}\left(\varepsilon^{1 / 2}, \mu^{1 / 2}\right)$. This order estimate can be understood by considering the case $\varepsilon=0$ (and $\mu>0$ ), sketched in fig. 5. Since the maximum of $R(r)$ is a quadratic one, the width of the maximum at the level 


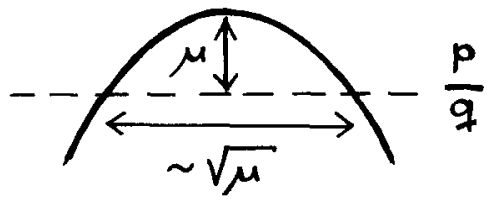

Fig. 5. The maximum of $R(r)$ for $\varepsilon=0$ and $\mu>0$. The width of the maximum at the level $p / q$ is proportional to $\sqrt{\mu}$, provided that the maximum is a quadratic one.

$p / q$ is proportional to $\mu^{1 / 2}$. This width is also equal to $2 x$, and therefore $x$ is $O\left(\mu^{1 / 2}\right)$. The non-uniform parts $\rho_{i}$ and $\psi_{i}$ are simply of order $\varepsilon$ or $\mu$, i.e. $\mathcal{O}(\varepsilon, \mu)$. For more details about these order estimates we refer to ref. [7]. Substituting expressions (15) in our model map (14) we get

$$
\begin{aligned}
& r_{0}+x+\rho_{i+1}=\frac{r_{0}+x+\rho_{i}}{1+\varepsilon g_{\varphi}\left(\varphi_{0}+2 \pi i p / q+\psi_{i}\right)}, \\
& \psi_{i+1}=\psi_{i}-2 \pi \beta\left(x+\rho_{i+1}\right)^{2}+\mu+\varepsilon g\left(\varphi_{0}+2 \pi i p / q+\psi_{i}\right) .
\end{aligned}
$$

The first equation (16a) may also be written as

$$
\begin{aligned}
\varepsilon g_{\varphi}\left(\varphi_{0}+2 \pi i p / q+\psi_{i}\right) & =\frac{r_{0}+x+\rho_{i}}{r_{0}+x+\rho_{i+1}}-1 \\
& -\frac{\rho_{i}-\rho_{i+1}}{r_{0}+x}+O\left(\varepsilon^{2}, \varepsilon \mu, \mu^{2}\right) .
\end{aligned}
$$

or, after summation over all the elements of the orbit,

$$
\varepsilon \sum_{i=1}^{4} g_{\psi}\left(\varphi_{0}+2 \pi i p / q+\psi_{i}\right)=0+O\left(\varepsilon^{2}, \varepsilon \mu, \mu^{2}\right)
$$

The second equation (16b) yields, after summation,

$$
\mu=2 \pi \beta x^{2}-\varepsilon \frac{1}{q} \sum_{i=1}^{q} g\left(\varphi_{0}+2 \pi i p / q+\psi_{i}\right)+O\left(\varepsilon^{2}, \varepsilon \mu, \mu^{2}\right)
$$

These two relations (18) and (19) govern the $p / q$ orbits, and we can directly determine the bifurcation diagram from them. We will show this for the specific choice

$$
g(\varphi)=\frac{1}{q} \cos (q \varphi)
$$

In this case condition (18) reads 


$$
\sum_{i=1}^{q} \sin \left[q\left(\varphi_{0}+2 \pi i p / q+\psi_{i}\right)\right]=0+\mathcal{O}(\varepsilon, \mu)
$$

which can be worked out to give

$$
\begin{aligned}
& \sum_{i=1}^{q}\left\{\sin \left[q\left(\varphi_{0}+2 \pi i p / q\right)\right] \cos \left(q \psi_{i}\right)+\cos \left[q\left(\varphi_{0}+2 \pi i p / q\right)\right] \sin \left(q \psi_{i}\right)\right\} \\
& \quad=\sum_{i=1}^{q} \sin \left[q\left(\varphi_{0}+2 \pi i p / q\right)\right]+\mathscr{O}(\varepsilon, \mu)=0+\mathcal{O}(\varepsilon, \mu)
\end{aligned}
$$

This implies that for all $i=1, \ldots, q$

$$
\sin \left[q\left(\varphi_{0}+2 \pi i p / q\right)\right]=0
$$

which tells us that the $p / q$ orbits bifurcate at the extrema of $g(\varphi)$. This is illustrated in fig. 6 . The first chain is born at the maxima: $\varphi=0(\bmod 2 \pi / q)$, and the second chain is born at the minima: $\varphi=\pi / q(\bmod 2 \pi / q)$. The values of $\mu$ at which these bifurcations take place are given by condition (19), which now reads

$$
\begin{aligned}
\mu & =2 \pi \beta x^{2}-\frac{\varepsilon}{q^{2}} \sum_{i=1}^{q} \cos \left[q\left(\varphi_{0}+2 \pi i p / q+\psi_{i}\right)\right] \\
& =2 \pi \beta x^{2}-\frac{\varepsilon}{q^{2}} \sum_{i=1}^{q} \cos \left[q\left(\varphi_{0}+2 \pi i p / q\right)\right]+\mathcal{O}\left(\varepsilon^{2}\right) \\
& =2 \pi \beta x^{2} \pm \frac{\varepsilon}{q^{2}}+\mathscr{O}\left(\varepsilon^{2}\right)
\end{aligned}
$$

where in the last step we have used eq. (23). The minus sign corresponds to the first chain, which bifurcates at the maxima of $q(\varphi)$, and the plus sign corresponds to the second chain, which bifurcates at the minima. From (24) we have

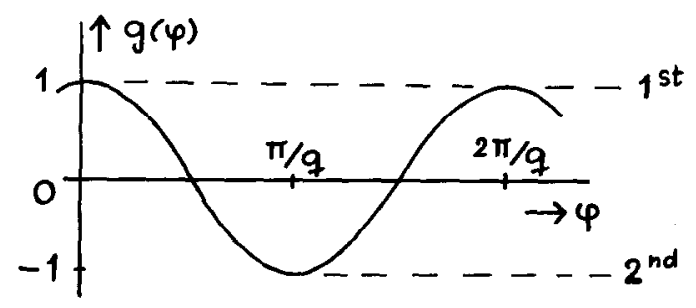

Fig. 6. The function $g(\varphi)=\cos (q \varphi) / q$ on its fundamental interval $0 \leqslant \varphi<2 \pi / q$. We have indicated the positions of the elements of the first and second chain by the dashed lines. 
for $x$, i.e. for the uniform part in the radial direction,

$$
x^{2}=\frac{\mu \mp \varepsilon / q}{2 \pi \beta}
$$

where the plus sign corresponds to the first chain, and the minus sign to the second. It follows immediately that the first chain bifurcates at $\mu=-\varepsilon / q$ (the above equation simply has no real solution for $\mu<-\varepsilon / q$ ), and the second chain at $\mu=\varepsilon / q$.

This is in perfect agreement with fig. 7 , where we show the bifurcation diagram for the case with $p / q=\frac{3}{10}$, obtained directly from computer plots of our model map (14). Indeed, we see that the bifurcation lines (enclosing the shaded cone) are $\varepsilon=-10 \mu$ and $\varepsilon=10 \mu$, respectively. And even more importantly, this diagram covers all the features of the standard scenario of figs. $3 \mathrm{~b}$ and 4. In fact, the "movie" for the Hénon map presented in fig. 4 corresponds to a roughly horizontal path through our bifurcation diagram 7 . This can be seen in fig. 8, where we show five computer plots for map (14), going from left to right through the bifurcation diagram at a fixed vlaue of $\varepsilon$ (namely $\varepsilon=0.0008$ ).

All this shows that map (14), with $g(\varphi)=(1 / q) \cos (q \varphi)$, is indeed an appropriate model for the standard scenario.

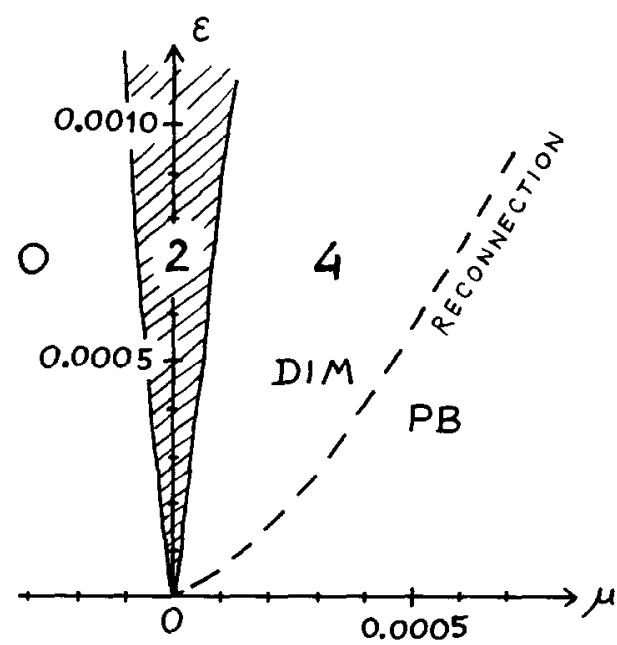

Fig. 7. The bifurcation diagram (numerically obtained) for the map (14), with $g(\varphi)$ given by (20), and $p / q=\frac{3}{10}$. Wc have indicated the regions with 0,2 and 4 periodic orbits. The region with 2 orbits (i.e. one dimerized chain) has been shaded; the boundaries of this region are $\varepsilon=-10 \mu$ and $\varepsilon=10 \mu$, respectively. The region with 4 orbits is divided in two subregions: in DIM the orbits form two dimerized chains, and in PB they form two Poincaré-Birkhoff chains. These two subregions are separated by the reconnection threshold (dashed line). 

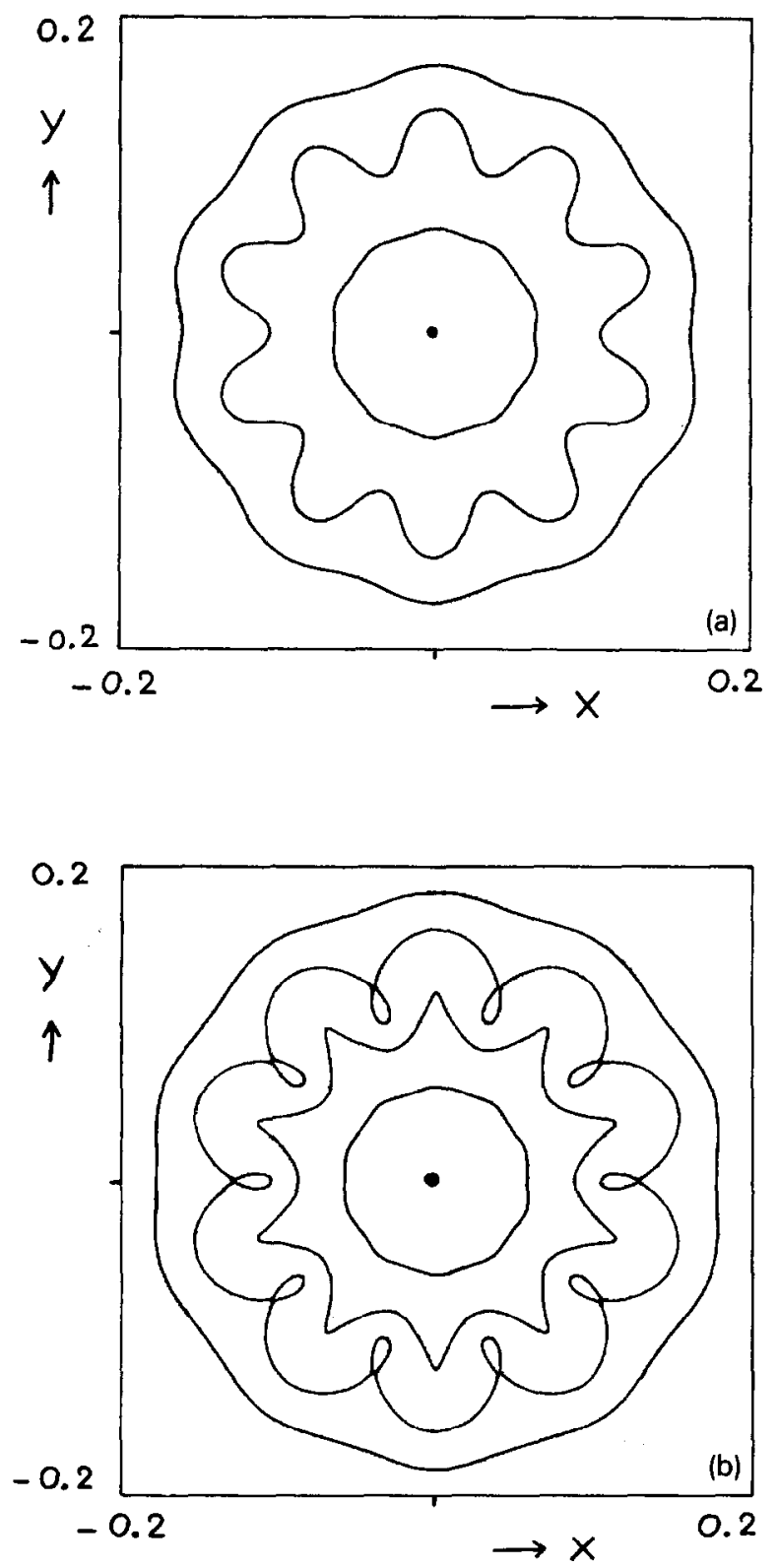

Fig. 8. Five computer plots of the $(x, y)$ plane for the map (14), with $g(\varphi)$ given by (20). These pictures correspond to a horizontal line through the bifurcation diagram 7 , at $\varepsilon=0.0008$. They should be compared to the five pictures in fig. 4 for the Hénon map. (a) At $\mu=-0.0004$ (in region 0 of fig. 7) prelude, (b) at $\mu=0$ (in region 2) one dimerized chain, (c) at $\mu=0.0003$ (in region 4 DIM) two dimerized chains, (d) at $\mu=0.00065$ (at the reconnection threshold), and (e) at $\mu=0.0010$ (in region $4 \mathrm{~PB}$ ) two $\mathrm{PB}$ chains. 

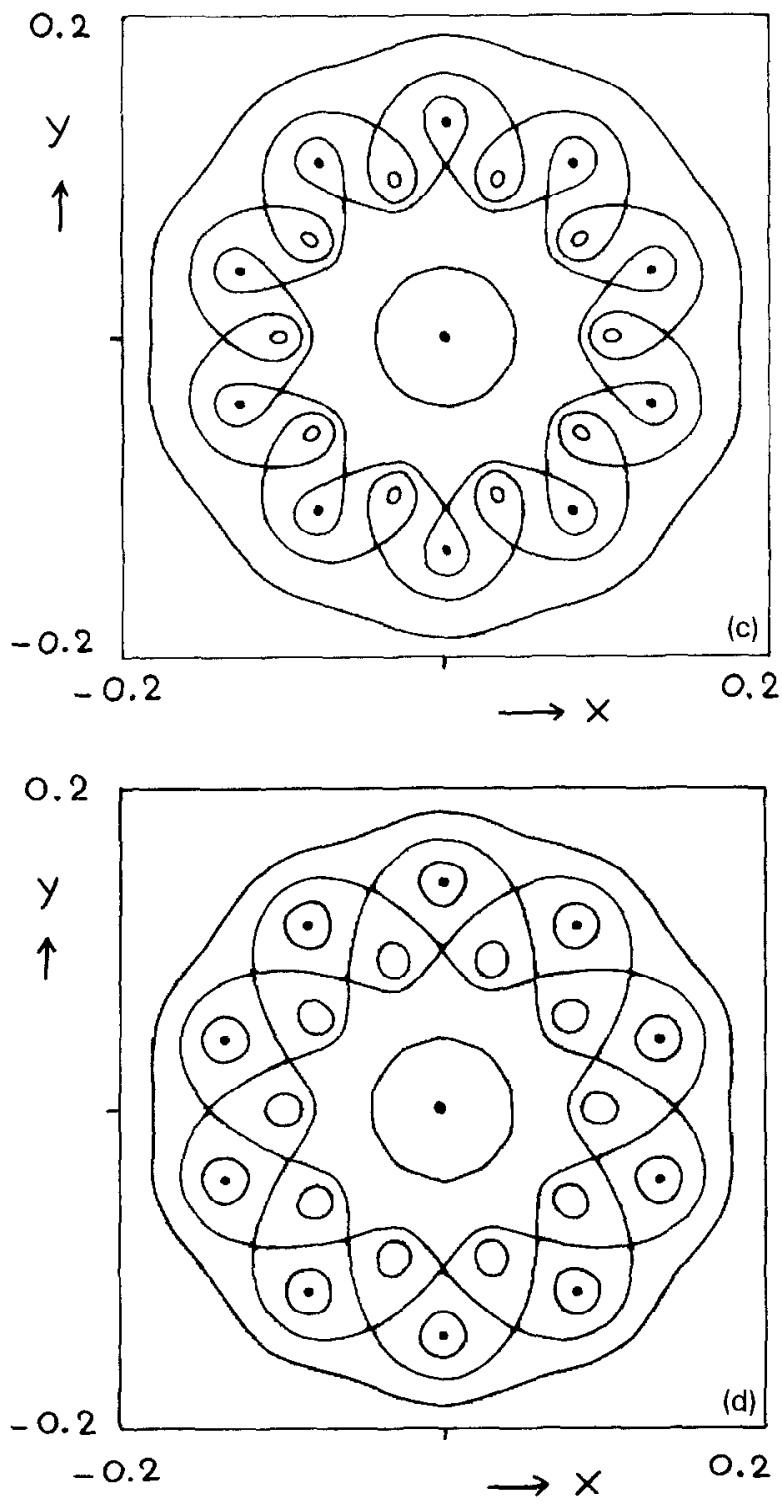

Fig. 8. (cont.).

In our bifurcation diagram 7 we see that the first dimerized chain of $p / q$ orbits is born already for negative $\mu$, i.e. before the maximum of $R(r)$ reaches the value $p / q$. This is of course due to the perturbing terms in the mapping. The effect is also clearly visible (once one knows what to look for) in figs. 4 a and $\mathrm{b}$ for the Hénon map, where $\rho(r)$ develops a peak reaching out to $p / q$ well before the smooth bulk gets there. 


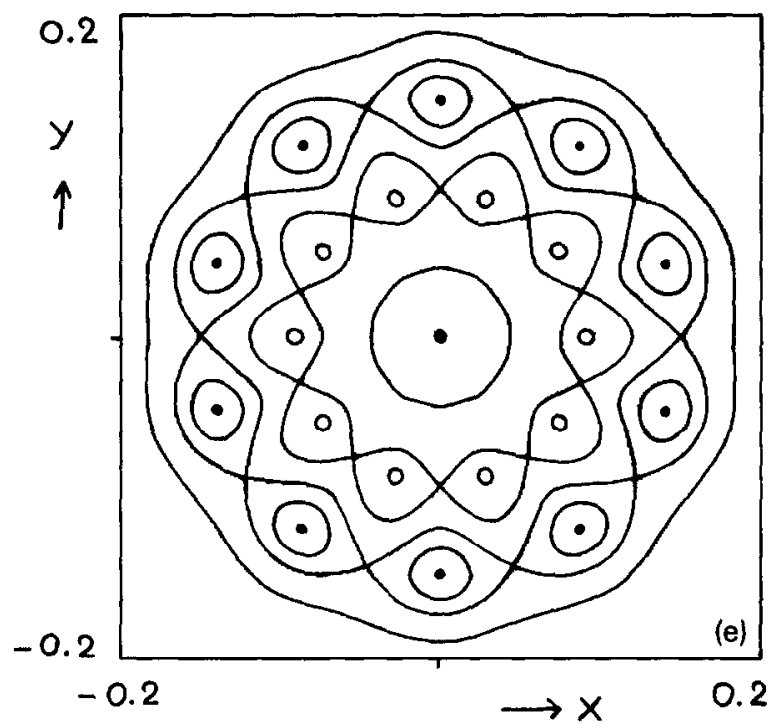

Fig. 8. (cont.).

We also see that the second dimerized chain is born for positive $\mu$, so this bifurcation is actually retarded by the perturbing terms in the map.

In the bifurcation diagram (fig. 7) we have also drawn the reconnection threshold, where the two dimerized chains are reconnected into two PB chains. The reconnection threshold is given by

$$
\varepsilon \approx C \mu^{3 / 2}
$$

where $C$ is a constant. This relation has been found by numerical inspection, and is in agreement with theoretical considerations given in ref. [3].

Our first object, to construct a good model for the standard scenario, has thus been accomplished. The above model gives us a clear insight in the anatomy of the standard scenario, and we may safely say we understand it now. We may also note that the standard scenario is the simplest scenario our model can possibly generate, since the functions $g(\varphi)$ and $F(x)$ which appear in this model were chosen as simple as possible. If we make other choices for $g(\varphi)$ and $F(x)$ we may anticipate more complicated scenarios. We will turn to this in the next section.

\section{A few comments}

\subsection{Another choice of $g(\varphi)$}

In this section we comment upon the generality of the results obtained so 
far. The standard scenario is arguably the most natural scenario, but certainly not the only one. In particular, we discuss what difference it would make if we made some other choices for the various functions in our model map (14).

First we consider another choice for the function $g(\varphi)$. Any periodic function with zero average and period $2 \pi / q$ will do. Let us take for instance the function depicted in fig. 9a,

$$
g(\varphi)=\frac{1}{q}\left[\cos (q \varphi)+\frac{1}{2} \cos (2 q \varphi)\right] .
$$

According to the condition (18) the $p / q$ orbits are born at angular positions $\varphi_{i}$ given by

$$
g_{\varphi}\left(\varphi_{i}\right)=-\left[1+2 \cos \left(q \varphi_{i}\right)\right] \sin \left(q \varphi_{i}\right)=0,
$$

that is, at the extrema of $g(\varphi)$. And according to condition (19) this happens at values of $\mu$ given by

$$
\mu=-\varepsilon g\left(\varphi_{i}\right) \text {. }
$$
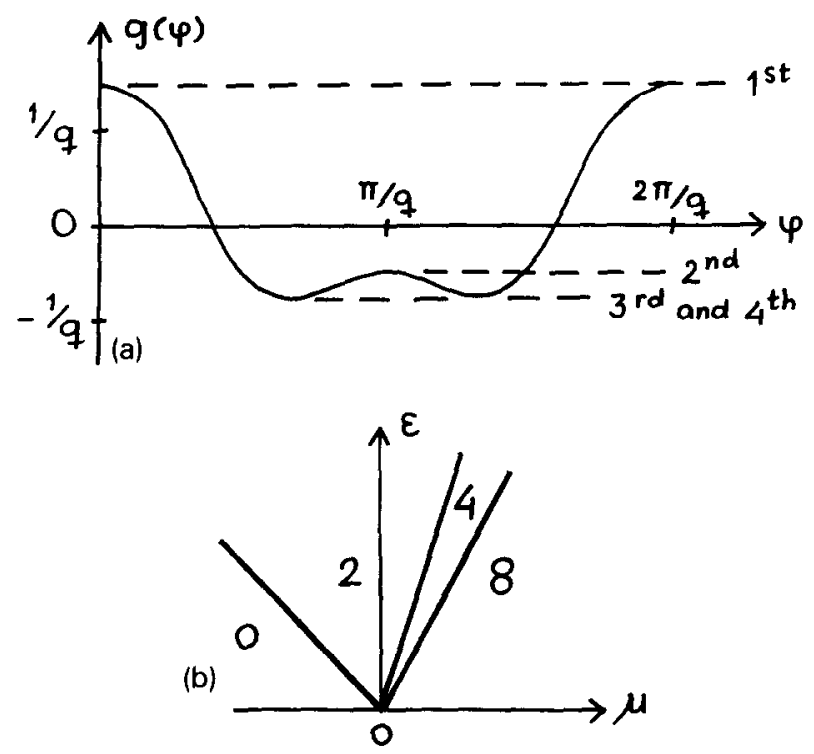

Fig. 9. (a) The function $g(\varphi)$ given by eq. (27), on the fundamental interval $0 \leqslant \varphi \leqslant 2 \pi / q$. The horizontal dashed lines indicate the births of the successive chains.

(b) The corresponding bifurcation diagram. The numbers in the different regions denote the number of $p / q$ orbits (which is twice the number of chains). The lines separating the regions are given by $\varepsilon=-\frac{2}{3} q \mu, \varepsilon=2 q \mu$ and $\varepsilon=\frac{4}{3} q \mu$, respectively, see also eq. (30). 
Now, the function $g(\varphi)$ has four extrema on the fundamental interval $0 \leqslant \varphi<2 \pi / q$, and accordingly we get four chains. This is indicated by the horizontal dashed lines in fig. 9a. The first chain is

$$
\varphi_{i}=0\left(\bmod \frac{2 \pi}{q}\right) \quad \text { at } \mu=-\frac{3}{2 q} \varepsilon,
$$

the second one is

$$
\varphi_{i}=\frac{\pi}{q}\left(\bmod \frac{2 \pi}{q}\right) \quad \text { at } \mu=\frac{1}{2 q} \varepsilon,
$$

and the third and fourth chains appear simultaneously,

$$
\begin{array}{ll}
\varphi_{i}=\frac{2 \pi}{3 q}\left(\bmod \frac{2 \pi}{q}\right) & \text { at } \mu=\frac{3}{4 q} \varepsilon, \\
\varphi_{i}=\frac{4 \pi}{3 q}\left(\bmod \frac{2 \pi}{q}\right) & \text { also at } \mu=\frac{3}{4 q} \varepsilon .
\end{array}
$$

The corresponding bifurcation diagram is given in fig. $9 \mathrm{~b}$. It is interesting to have a look at the birth process in this case, including the reconnection phenomenon. Although in essence it is similar to the process for two chains, it is more intricate due to the fact that we have twice as many fixed points. The different stages are schematically drawn in fig. 10.

Fig. 10 is based on real-life computerplots, of which we show one example in fig. 11. This particular plot corresponds to stage $\mathrm{E}$ in fig. 10 , with $p / q=\frac{1}{5}$. It contains eight orbits of period 5 , four hyperbolic ones (consisting of 5 saddle points each) and four elliptic ones (consisting of 5 center points each). At first glance it might be tempting to believe (especially for those who are familiar with pictures of period-doubling bifurcations) that the center points contained within the dumb-bells together form one orbit of period 10 , but this is not the case. The white and black points form two distinct orbits of period 5. The delusion is simply due to the symmetry of the two orbits (which are born simultaneously, see eqs. (30c) and (30d)).

One of the beautiful features of this picture is that we are looking at a half-completed reconnection. The innermost dimerized chain is still intact, while the outermost orbits have already taken the shape of a PoincaréBirkhoff chain. In between, the dumb-bells (with their horizontal PB-type connections) are still surrounded by a dimer-type heteroclinic curve. It is a truly hybrid picture, with dimerized and PB characteristics going hand in hand.

The above example shows how easy it is to construct variations on the standard scenario. Depending on the choise of the $g(\varphi)$ one can make these 

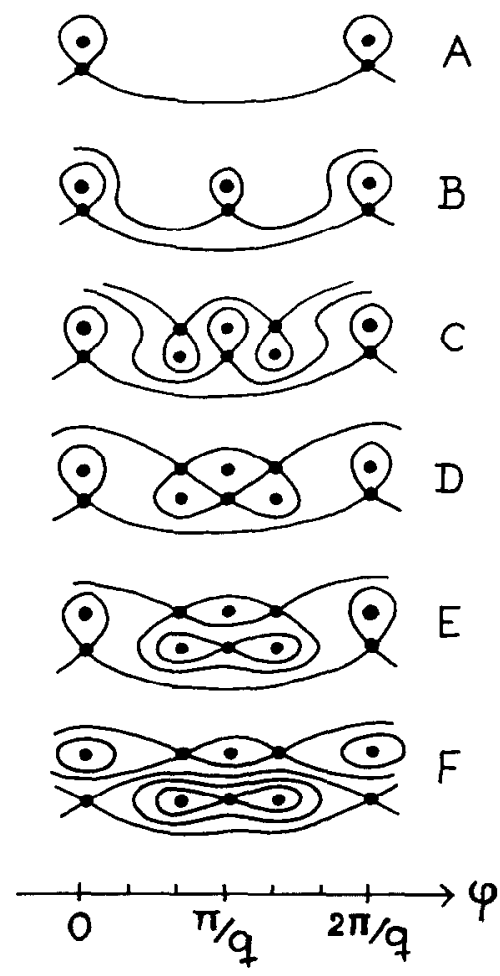

Fig. 10. Six successive stages in the birth and reconnection process of the periodic orbits for the map (14), with $g(\varphi)$ given by (27). Stage A corresponds to the region indicated by 2 in tig. $9 \mathrm{~b}$. stage $\mathrm{B}$ corresponds to the region indicated by 4 , and the stages $\mathrm{C}, \mathrm{D}, \mathrm{E}, \mathrm{F}$ all take place in the region indicated by 8 .

variations as wild as one wishes and the resulting pictures may become very aesthetic, sometimes forming whole "webs" of intertwined chains, dimerized and PB-type chains alike. However, the pictures also become quite artificial and we would be surprised if they were found in a real physical situation.

\subsection{Another choice of $F(x)$}

I.et us next consider another choice of the function $F(x)$ given by eq. (13). Up to now we have taken $F(x)=1$, which is the simplest choice possible, so let us now take the next simplest choice $F(x)=x$. That is,

$$
\int_{0}^{r^{\prime}} F(x) \mathrm{d} x=\frac{1}{2} r^{\prime 2} .
$$




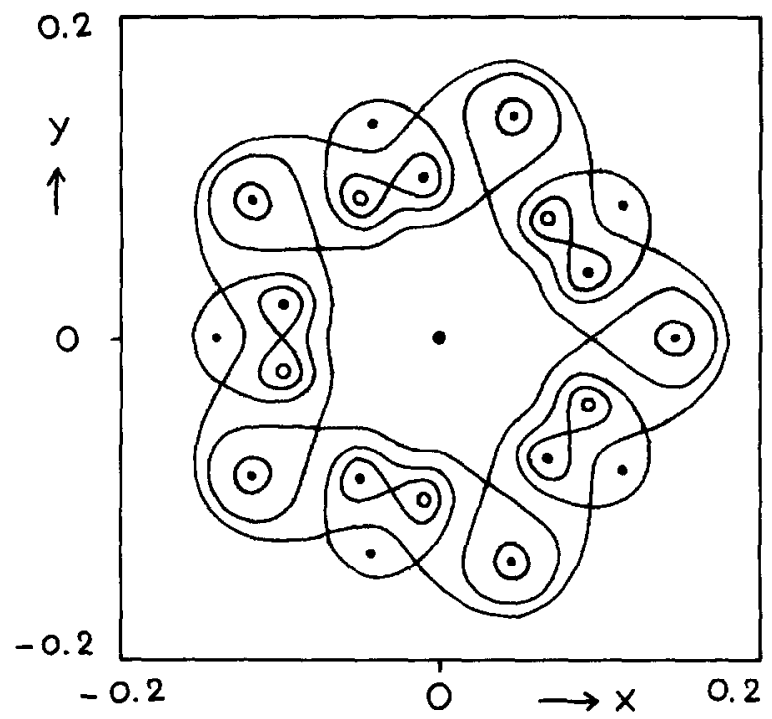

Fig. 11. A computer picture for the map (14), with $g(\varphi)$ as in (27) and $p / q=\frac{1}{5}$. This picture corresponds to stage $\mathrm{E}$ in fig. 10. It contains (apart from the fixed point in the origin) four stable and four unstable orbits of period 5 .

In that case we get from the generating function

$$
\begin{aligned}
& r=r^{\prime}+\frac{1}{2} \varepsilon r^{\prime 2} g_{\varphi}(\varphi), \\
& \varphi^{\prime}=\varphi+2 \pi\left(\frac{p}{q}-\beta\left(r^{\prime}-r_{0}\right)^{2}\right)+\mu+\varepsilon r^{\prime} g(\varphi) .
\end{aligned}
$$

To invert (32a) we regard it as a quadratic equation of $r^{\prime}$, which yields

$$
r^{\prime}=\frac{1}{\varepsilon g_{\varphi}(\varphi)}\left[-1 \pm \sqrt{1+2 \varepsilon r g_{\varphi}(\varphi)}\right]
$$

Retaining the solution with the plus sign (since $r^{\prime}$ must be positive), and writing out the Taylor series expansion of the square root, we get

$$
r^{\prime}=r\left[1-\frac{1}{4} \operatorname{Erg}_{\varphi}(\varphi)+\mathcal{O}\left(\varepsilon^{2}\right)\right] \text {, }
$$

which should be compared with the expression in (14),

$$
r^{\prime}=\frac{r}{1+\varepsilon g_{\varphi}(\varphi)}=r\left[1-\varepsilon g_{\varphi}(\varphi)+\mathcal{O}\left(\varepsilon^{2}\right)\right]
$$


We see that the choice (31) has introduced an extra $r$-dependence in the perturbing term of the map. This makes the map somewhat more general, in the sense that the inner and outer chains will no longer be equivalent. As long as the map is area-preserving this has no qualitative effect on the bifurcation diagram, but if we add a dissipation to our map then the difference becomes important, as we shall see in the next section.

\section{The influence of dissipation}

\subsection{Adding a dissipation to our model}

Up to now we have been talking about conservative systems, without any dissipation. This is all very well, but in real life one rarely encounters a system which has no dissipation at all. Therefore, in this section we want to investigate the effect of (a slight amount of) dissipation on the birth process. As a starting point we take again the model map (14), and we add a dissipation to this map in the following simple way:

$$
\left\{\begin{array}{l}
r^{\prime}=\frac{(1-\delta) r}{1+\varepsilon g_{\varphi}(\varphi)}, \\
\varphi^{\prime}=\varphi+2 \pi\left(\frac{p}{q}-\beta\left(r^{\prime}-r_{0}\right)^{2}\right)+\mu+\varepsilon g(\varphi) .
\end{array}\right.
$$

We have introduced a third parameter $\delta$, which is a measure of the strength of the dissipation. The map is now area-contracting, with Jacobian $1-\delta$, and as a consequence all the stable fixed points (which in the conservative case were centers) now become attracting spiral points.

Inserting the expression (15) for the bifurcating $p / q$ orbit into the above map we get

$$
\begin{aligned}
& r_{0}+x+\rho_{i+1}=\frac{(1-\delta)\left(r_{0}+x+\rho_{i}\right)}{1+\varepsilon g_{\varphi}\left(\varphi_{0}+2 \pi i p / q+\psi_{i}\right)}, \\
& \psi_{i+1}=\psi_{i}-2 \pi \beta\left(x+\rho_{i+1}\right)^{2}+\mu+\varepsilon g\left(\varphi_{0}+2 \pi i p / q+\psi_{i}\right),
\end{aligned}
$$

and this yields the following two conditions (up to leading order in the parameters $\varepsilon, \mu$ and $\delta$ ):

$$
\begin{aligned}
& \delta=-\frac{\varepsilon}{q} \sum_{i=1}^{q} g_{\varphi}\left(\varphi_{0}+2 \pi i p / q+\psi_{i}\right), \\
& \mu=2 \pi \beta x^{2}-\frac{\varepsilon}{q} \sum_{i=1}^{g} g\left(\varphi_{0}+2 \pi i p / q+\psi_{i}\right) .
\end{aligned}
$$


Note that the second condition still has the same form as in the case without dissipation, but the first condition has changed. In fact, this condition tells us that if $\delta$ becomes too large $\left(\delta \geqslant \max \left|\varepsilon g_{\varphi}\right|\right)$ we do not get any $p / q$ chains at all.

To discuss this in some more detail we must take a specific function $g(\varphi)$. Taking $g(\varphi)=(1 / q) \cos (q \varphi)$ as before, the two conditions become (after the usual manipulations, cf. eq. (22))

$$
\begin{aligned}
& \delta=\frac{\varepsilon}{q} \sum_{i=1}^{q} \sin \left[q\left(\varphi_{0}+2 \pi i p / q\right)\right], \\
& \mu=2 \pi \beta x^{2}-\frac{\varepsilon}{q^{2}} \sum_{i=1}^{q} \cos \left[q\left(\varphi_{0}+2 \pi i p / q\right)\right] .
\end{aligned}
$$

Taking the first of these two equations and working it out like before, we get for all $i=1,2, \ldots, q$

$$
\varepsilon \sin \left[q\left(\varphi_{0}+2 \pi i p / q\right)\right]=\delta,
$$

which should be compared with eq. (23). This relation is illustrated in fig. 12 and it tells us that for finite $\delta$ the $p / q$ orbits no longer bifurcate at the extrema of $g(\varphi)$, i.e. at $\varphi=0(\bmod 2 \pi / q)$ and $\varphi=\pi / q(\bmod 2 \pi / q)$, but somewhere in between. We see that for increasing $\delta$ the elements of the two chains approach each other pairwise, until for $\delta=\varepsilon$ they coincide at $\varphi=\pi / 2 q(\bmod \pi / q)$. There they annihilate each other since for $\delta>\varepsilon$ no $p / q$ orbit can exist anymore.

The values of $\mu$ at which the respective bifurcations of the two chains take place are subsequently determined by condition (39b). In fact, from (39a) and (39b) together we can infer the whole bifurcation diagram. To this end, we write them in the following form:

$$
f\left(x, \varphi_{0}\right)-\varepsilon \sum_{i=1}^{q} \sin \left[q\left(\varphi_{0}+2 \pi i p / q\right)\right]-\delta q=0,
$$

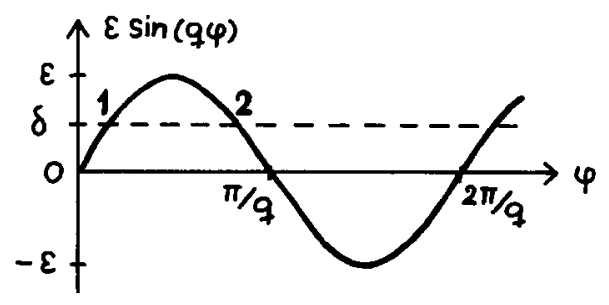

Fig. 12. Illustration of eq. (40) on the fundamental interval $0 \leqslant \varphi<2 \pi / q$, showing the position of two elements of the first and second chain (indicated by 1 and 2). 


$$
h\left(x, \varphi_{0}\right)=2 \pi \beta x^{2}-\mu-\frac{\varepsilon}{q^{2}} \sum_{i=1}^{q} \cos \left[q\left(\varphi_{0}+2 \pi i p / q\right)\right]=0 .
$$

If the determinant $(\partial f / \partial x)\left(\partial h / \partial \varphi_{0}\right)-(\partial h / \partial x)\left(\partial f / \partial \varphi_{0}\right)$ is unequal zero then, according to the implicit function theorem, these two equations have a unique solution for small values of $\varepsilon, \mu$ and $\delta$. A necessary condition for a bifurcation (when there must be at least two solutions, and therefore no uniqueness) is therefore

$$
\left|\begin{array}{ll}
\frac{\partial f}{\partial x} & \frac{\partial f}{\partial \varphi_{0}} \\
\frac{\partial h}{\partial x} & \frac{\partial h}{\partial \varphi_{0}}
\end{array}\right|=-4 \pi \beta x q \sum_{i=1}^{q} \cos \left[q\left(\varphi_{0}+2 \pi i p / q\right)\right]=0 .
$$

This then should be regarded as the bifurcation condition. That is, at bifurcation we have either

$$
x=0
$$

or

$$
\sum_{i=1}^{q} \cos \left[q\left(\varphi_{0}+2 \pi i p / q\right)\right]=0
$$

In the case $x=0$ we get from (41b)

$$
\mu=-\frac{\varepsilon}{q^{2}} \sum_{i=1}^{q} \cos \left[q\left(\varphi_{0}+2 \pi i p / q\right)\right]
$$

without any restriction on $\varphi_{0}$. There are two limiting cases here;

i) For $\cos \left[q\left(\varphi_{0}+2 \pi i p / q\right)\right]=0$ we get $\mu=0$, and also $\sin \left[q\left(\varphi_{0}+2 \pi i p / q\right)\right]=$ \pm 1 and consequently (from eq. (41a)) $\varepsilon= \pm \delta$. Since we have chosen to take $\varepsilon$ and $\delta$ both positive, we keep only the plus solution.

ii) For $\cos \left[q\left(\varphi_{0}+2 \pi i p / q\right)\right]= \pm 1$ we get $\mu=\mp \varepsilon / q$, and (from eq. (41a) with $\left.\sin \left[q\left(\varphi_{0}+2 \pi i p / q\right)\right] \rightarrow 0\right) \varepsilon \gg \delta$. This limit is just like the non-dissipative case treated in section 4 .

Interpolating between these two limiting cases we see that eq. (44) gives rise to a bifurcation line in the $(\varepsilon, \mu)$ plane in the form of a blunt, vertical cone, which has its tip at $\mu=0$ for $\varepsilon=\delta$ and which approximates the lines $\mu= \pm \varepsilon / q$ for $\varepsilon \gg \delta$. This blunt cone can easily be recognized in the diagram of fig. 13 .

The second bifurcation line follows from (43b). In this case we get from (41b) that $\mu=2 \pi \beta x^{2}$, so the only restriction on $\mu$ is that it should be 


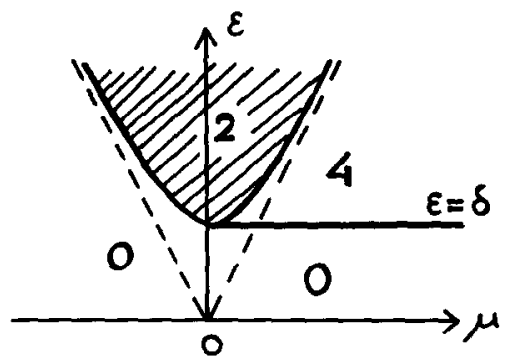

Fig. 13. The bifurcation diagram for the map (36). The dashed lines, given by $\varepsilon= \pm q \mu$, represent the non-dissipative case $(\delta=0)$ and are simply meant as a guide to the eye.

non-negative. And (41a) now reads, since $\sin \left[q\left(\varphi_{0}+2 \pi i p / q\right)\right]= \pm 1$,

$$
\varepsilon= \pm \delta \text {. }
$$

Again, since we consider only positive values of $\varepsilon$ and $\delta$ we keep only the plus solution, and thus we get in the $(\varepsilon, \mu)$ plane a horizontal line $\varepsilon=\delta$ for all values $\mu \geqslant 0$. This second bifurcation line completes the diagram shown in fig. 13.

At this point we stop for a moment and take a close look at our bifurcation diagram. In particular, we consider the horizontal line $\varepsilon=\delta$. Here the saddles and nodes of the outer two $p / q$ orbits annihilate each other pairwise, and simultaneously the saddles and nodes of the inner two orbits do the same. This simultaneousness can hardly be generic, and it must be due to a superfluous symmetry in our model map. It is not difficult to guess that this symmetry lies in the fact that the $\varepsilon$-terms in our map (36), that is $\varepsilon g_{\varphi}(\varphi)$ and $\varepsilon g(\varphi)$, do not depend on the radius. From the previous section, however, we already know how to cure this.

\subsection{Curing the non-genericity}

In eqs. (32)-(34) we have already introduced an adapted version of our model map including an $r$ dependence in the $\varepsilon$-terms. This map reads, with dissipation,

$$
\left\{\begin{array}{l}
r^{\prime}=(1-\delta) r\left[1-\frac{1}{4} \varepsilon r g_{\varphi}(\varphi)\right], \\
\varphi^{\prime}=\varphi+2 \pi\left(\frac{p}{q}-\beta\left(r^{\prime}-r_{0}\right)^{2}\right)+\mu+\varepsilon r^{\prime} g(\varphi),
\end{array}\right.
$$

and the two conditions for the $p / q$ orbits take the following form, up to leading orders in the parameters $\varepsilon, \mu$ and $\delta$ :

$$
\delta=-\frac{\varepsilon}{4 q}\left(r_{0}+x\right) \sum_{i=1}^{q} g_{\varphi}\left(\varphi_{0}+2 \pi i p / q+\psi_{i}\right),
$$




$$
\mu=2 \pi \beta x^{2}-\frac{\varepsilon}{q}\left(r_{0}+x\right) \sum_{i=1}^{q} g\left(\varphi_{0}+2 \pi i p / q+\psi_{i}\right),
$$

which should be compared with eqs. $(38 \mathrm{a}, \mathrm{b})$. For $g(\varphi)=(1 / q) \cos (q \varphi)$ these conditions become

$$
\begin{aligned}
& f\left(x, \varphi_{0}\right)=\varepsilon\left(r_{0}+x\right) \sum_{i=1}^{q} \sin \left[q\left(\varphi_{0}+2 \pi i p / q\right)\right]-4 q \delta=0 \\
& h\left(x, \varphi_{0}\right)=2 \pi \beta x^{2}-\mu-\frac{\varepsilon}{q^{2}}\left(r_{0}+x\right) \sum_{i=1}^{q} \cos \left[q\left(\varphi_{0}+2 \pi i p / q\right)\right]=0 .
\end{aligned}
$$

The first of these two equations can be worked out, as before, to give

$$
\delta=-\frac{\varepsilon}{4}\left(r_{0}+x\right) \sin \left[q\left(\varphi_{0}+2 \pi i p / q\right)\right]
$$

for all $i=1,2, \ldots, q$. This relation (which should be compared with eqs. (23) and $(40)$ ) is illustrated in fig. 14, at two fixed values of $x$. The solid curve corresponds to the outer $p / q$ orbits, with $x>0$, and the dashed curve corresponds to the inner $p / q$ orbits, with $x<0$.

Thus we see that our new map (46) does indeed discriminate between the inner and the outer orbits. The inner elements of the bifurcating chains, denoted by 1 ' and 2 ', lie closer to each other (pairwise) than the outer elements denoted by 1 and 2 . For $\delta=0$ the difference vanishes, and that is why in the non-dissipative case we never even had to think about it. But for increasing $\delta$ the difference becomes more and more pronounced, until the inner orbits meet (and annihilate each other) while the outer orbits are still apart and alive. They

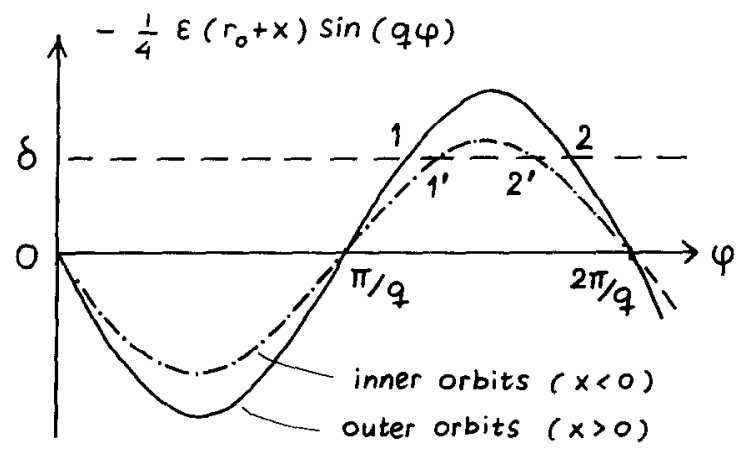

Fig. 14. Illustration of eq. (49), for the outer orbits as well as for the inner orbits. We see that only for $\delta=0$ the $\varphi$-values of the inner and outer orbits are the same; for growing $\delta$ the difference increases. The elements of the inner orbits $1^{\prime}$ and $2^{\prime}$ annihilate each other at a smaller value of $\delta$ than the outer orbits 1 and 2 . 
meet (and annihilate) each other at a higher value of $\delta$. From this one can already understand that the former bifurcation line $\varepsilon=\delta$ has been split into two, just as we wanted.

To make this a little bit more explicit (see also ref. [7] for related considerations) we consider conditions $(48 \mathrm{a}, \mathrm{b})$. For bifurcation we require, as before, that the determinant is zero:

$$
\frac{\partial f}{\partial x} \frac{\partial h}{\partial \varphi_{0}}-\frac{\partial h}{\partial x} \frac{\partial f}{\partial \varphi_{0}}=0
$$

That is,

$$
\begin{aligned}
& \frac{\varepsilon^{2}}{q}\left(r_{0}+x\right)\left(\sum_{i=1}^{q} \sin \left[q\left(\varphi_{0}+2 \pi i p / q\right)\right]\right)^{2} \\
& \quad+\left(\frac{\varepsilon^{2}}{q}-4 \pi \beta q \varepsilon x\right)\left(r_{0}+x\right) \sum_{i=1}^{q} \cos \left[q\left(\varphi_{0}+2 \pi i p / q\right)\right]=0 .
\end{aligned}
$$

The leading term here is

$$
-4 \pi \beta q \varepsilon x r_{0} \sum_{i=1}^{q} \cos \left[q\left(\varphi_{0}+2 \pi i p / q\right)\right],
$$

which is of order $\frac{3}{2}$ in the small parameters (since $x$ is of order $\varepsilon^{1 / 2}$ as demonstrated in section 4). This term alone would give exactly the same conditions as before, namely eqs. $(43 a, b)$, and qualitatively also the same bifurcation diagram. However, this diagram is only approximate in the present case, since the next important terms (which are of order 2 in the small parameters):

$$
\begin{aligned}
& \frac{\varepsilon^{2}}{q} r_{0}\left(\sum_{i=1}^{q} \sin \left[q\left(\varphi_{0}+2 \pi i p / q\right)\right]\right)^{2} \\
& \quad+\left(\frac{\varepsilon^{2}}{q} r_{0}-4 \pi \beta q \varepsilon x^{2}\right) \sum_{i=1}^{q} \cos \left[q\left(\varphi_{0}+2 \pi i p / q\right)\right],
\end{aligned}
$$

change the position of the bifurcation lines. The change may only be small, but it is important all the same. In particular, the horizontal bifurcation line which would lie at $\varepsilon=4 \delta / r_{0}$ if it were for the leading term (52) alone, is now split into two. Thus the map (46), which was actually constructed in order to achieve this splitting, has done exactly what we wanted it to do.

The resulting bifurcation diagram has been sketched in fig. 15. Those who are familiar with the so-called elementary catastrophes may recognize in this picture a generic cut through a swallowtail catastrophe [8]. Along with this diagram, it is illuminating to show a few plots of the map (46) at different values of the parameters $\varepsilon$ and $\mu$; in fact, we show a movie for a whole series 


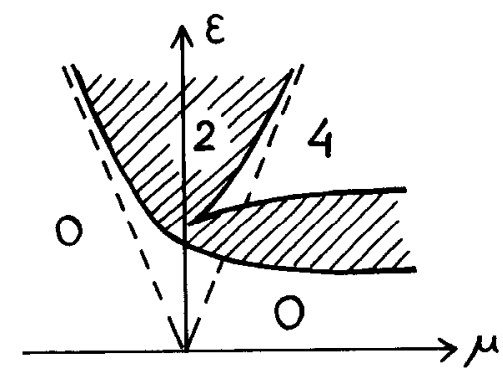

Fig. 15. The bifurcation diagram for the map (46). The dashed lines, given by $\varepsilon= \pm q \mu$, represent the non-dissipative case $(\delta=0)$ and are meant as guide to the eye.

of $(\varepsilon, \mu)$ values as indicated in fig. 16a, with $\delta=0.0001$ fixed. The movie (slightly schematized for clarity) is presented in fig. 16b. As anticipated, we see that the outer orbits are more persistent than the inner ones. The inner saddle-node pair is already gone in fig. $16 \mathrm{~b}$, stage $H$, while the outer pair is still alive. It is also interesting to note that the reconnection now occurs in three steps. In stage D we have two saddles and two nodes which are grouped in two radial pairs, and via stage $E$ these are reconnected into the two tangential pairs shown in stage $F$. This unfolding of the reconnection threshold
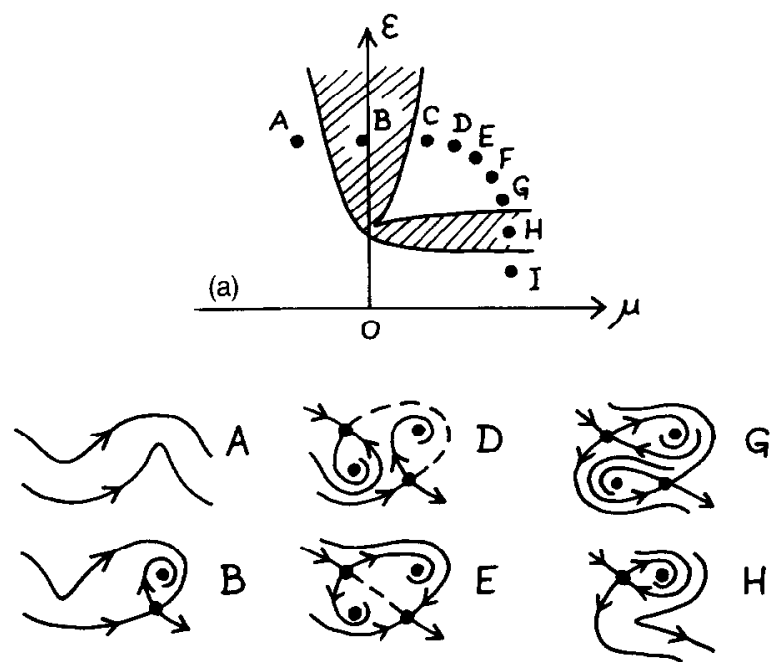

(b)
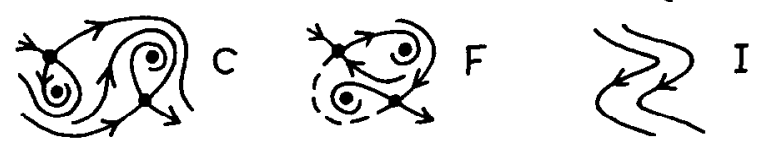

Fig. 16. A schematic representation of the birth $(A, B, C)$, the reconnection $(D, E, F)$ and the annihilation $(\mathrm{G}, \mathrm{H}, \mathrm{I})$ of periodic orbits for the map $(46)$, with $g(\varphi)=\cos (q \varphi) / q$. The pictures correspond to a fixed value of $\delta>0$, and $\varepsilon$ and $\mu$ as indicated in the bifurcation diagram. 
into three lines is quite understandable. In the area-preserving case all the saddle-saddle connections between the inner and the outer orbits were equivalent and therefore they were all reconnected at once, in one single step, but in the presence of dissipation this equivalence among the saddle connections is lost, and hence they are reconnected one by one.

\subsection{The Hénon map with dissipation}

In order to check the general validity of the above results we now consider an example from real life, the Hénon map, with a dissipation added to it:

$$
\left\{\begin{array}{l}
x_{n+1}=2 C x_{n}+2 x_{n}^{2}-y_{n}, \\
y_{n+1}=(1-\delta) x_{n} .
\end{array}\right.
$$

From section 2 we already know that for $C=-0.306294$ and $\delta=0$ this map has four $\frac{3}{10}$ orbits, grouped in two dimerized chains. If we now increase $\delta$ we expect several changes to occur, according to the scenario found in section 6.2. In the first place the "dimers" should move towards each other pairwise. In the second place, the saddles and nodes should be reconnected from radially oriented saddle-node pairs into horizontal pairs. After that, the inner pairs should vanish by a saddle-node annihilation, and eventually the outer pairs should vanish too.

In fig. 17, where we show four plots for increasing values of $\delta$ we see that all our expectations come true. This (together with the common notion that the Hénon map is as typical as can be) gives further ground to our statement that the model map (46), with the choices we have made there for the functions $R(r), F(r)$ and $g(\varphi)$, indeed is a typical one and the birth process for this map can be regarded as the standard scenario in the presence of dissipation.

As a side remark, it is interesting to note the smallness of $\delta$ at which the annihilations occur. The first annihilation occurs at $\delta \approx 3.5 \times 10^{-6}$, and the second at $\delta \approx 5.5 \times 10^{-6}$. This means that the Hénon map corresponds to a model with a very small parameter $\varepsilon$. Indeed, if we use our model map (46), for which these annihilations are centered around the horizontal line $\varepsilon=4 \delta_{\text {mean }} / r_{0}$, the above values of $\delta$ (with $\delta_{\text {mean }} \approx 4.5 \times 10^{-6}$ ) would imply that

$$
\varepsilon=\frac{4 \delta_{\text {mean }}}{r_{0}} \approx \frac{4 \times 4.5 \times 10^{-6}}{0.11}=1.6 \times 10^{-4} .
$$

From this one may conclude that the deviation from integrability in the Hénon map is only very, very small. 

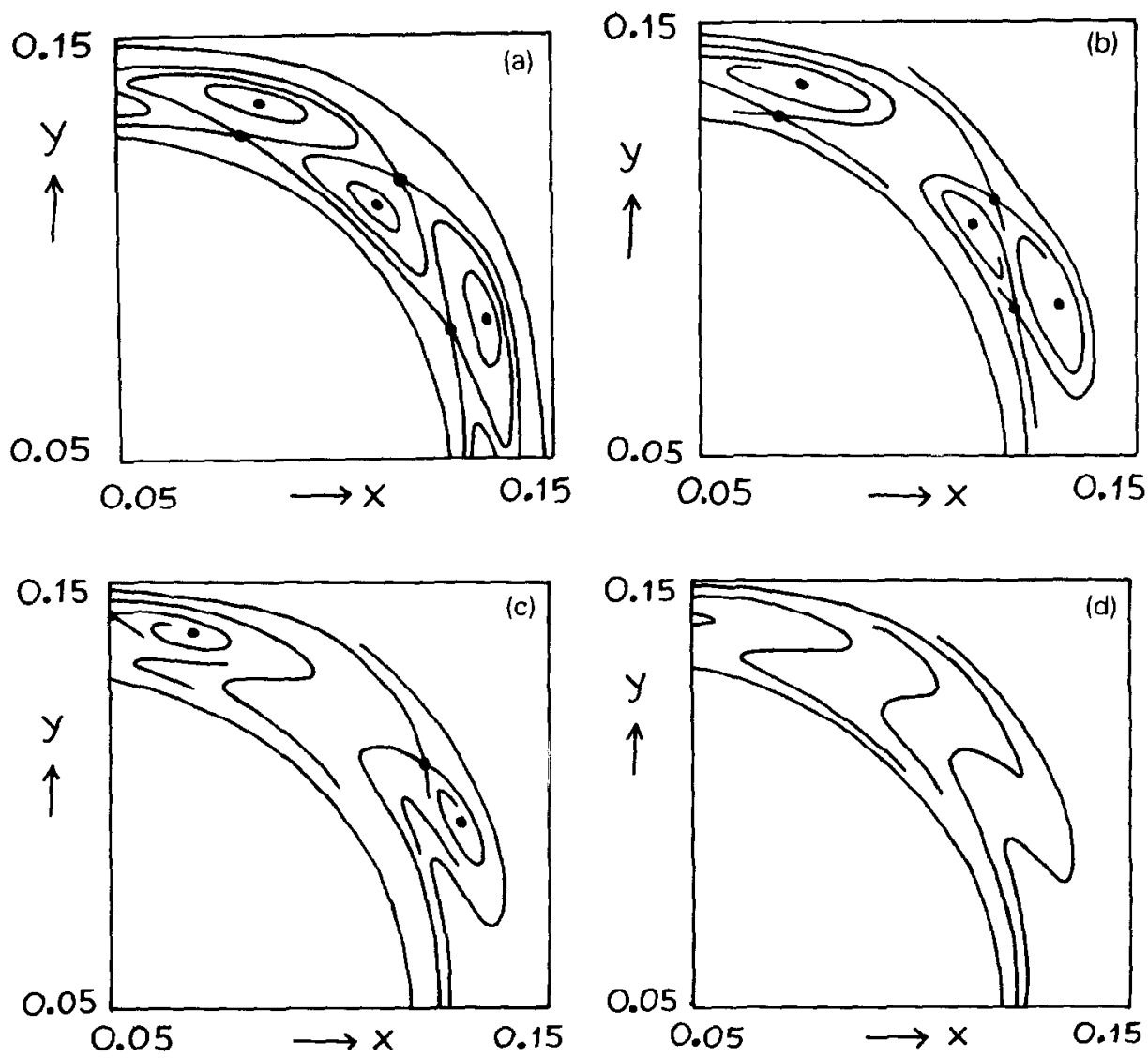

Fig. 17. (a) Detail of the $(x, y)$ plane for the Hénon map (54), with $C=-0.306294$ and $\delta=0$, i.e. the area-preserving case. This picture would fit in the movie of fig. 4 between the stages (b) and (c).

(b) The same region as in (a), but now with $\delta=2 \times 10^{-6}$. We still have four $\frac{3}{10}$ orbits, but the influence of the dissipation is clearly visible.

(c) The same region as in (a) and (b) but now with $\delta-4 \times 10^{\circ}$. We see that the inner orbits have annihilated each other, and only the outer two $\frac{3}{10}$ orbits are left.

(d) The same region as in (a)-(c) but now with $\delta=6 \times 10^{-6}$. Also the outer two orbits have annihilated each other.

\section{Discussion}

This paper has clarified, we hope, the birth of periodic orbits in non-twist maps (near an extremum of the rotation number). In retrospect we can say that the key to understanding the birth process has been the model map introduced in section 3 .

In its simplest form this model map proved to reproduce the birth process as 
we knew it from the area-preserving Hénon map, which we called the standard scenario. The corresponding bifurcation diagram (see fig. 7) has been calculated in section 4.

Variations on the standard scenario are also possible within the context of our model map, and in section 5 we have illustrated this with an example. These variations can give rise to beautiful "webs" of periodic orbits tangled together (these webs are related to those of ref. [9]), but they are certainly not typical, and in practical situations we expect the standard scenario to be the prevailing form. Experimental results, such as the surface of section plots for the beam-beam interaction in ref. [10], confirm this expectation.

Another kind of variation on the standard scenario was discussed in section 6 , where we described the effects of dissipation. This is of considerable practical interest, since in real experiments one will often have to face at least a small amount of dissipation. Our main results are summed up in fig. 16.

In our calculations we have always taken the perturbation parameter $\varepsilon$ to be non-negative, which can indeed be done without loss of generality. Nevertheless, it may be instructive to let this restriction go for once and sketch the bifurcation diagrams in the complete $(\varepsilon, \mu)$ plane. In fig. 18a we have done this for the conservative case, and in fig. $18 \mathrm{~b}$ for the dissipative case. These diagrams show how the dissipation "eats" a tunnel through the regions 2 and 4 .

As a final remark, we would like to point out once more (see also refs. [1, 5, 11]) that a non-twist situation is by no means exceptional. In fact, it occurs generically in Hamiltonian and near-Hamiltonian systems near 1:3 resonance of an elliptic fixed point (i.e. when the rotation number around this point is close to $\frac{1}{3}$ ) and sometimes near the 1:4 resonance as well. Thus, the results derived in this paper should apply to a wide variety of physical situations.
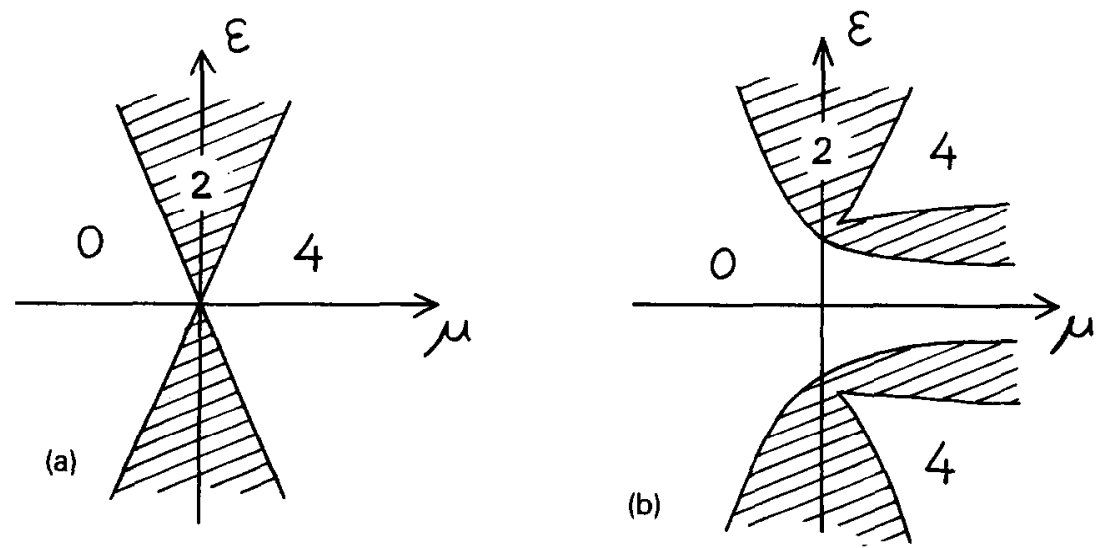

Fig. 18. The generic bifurcation diagrams for the conservative (a) as well as for the dissipative case (b), in the full $(\varepsilon, \mu)$ plane, i.e. without our earlier restriction $\varepsilon \geqslant 0$. 


\section{Acknowledgements}

One of the authors (JPvdW) would like to thank R.S. MacKay for several very stimulating discussions during a visity to Warwick University. We are also grateful to H.W. Capel and T. Post from the University of Amsterdam for their keen interest and valuable comments.

\section{References}

[1] J.P. van der Weele, T.P. Valkering, H.W. Capel and T. Post, Physica A 153 (1988) 283. See also: J.P. van der Weele, in: Proc. Int. Conf. on Singular Behaviour and Nonlinear Dynamics 1988, Samos, Greece, St. Pnevmatikos, T. Bountis and Sp. Pnevmatikos, eds. (World Scientific, Singapore, 1989) vol. 1, p. 118.

[2] R.S. MacKay and J.D. Meiss, eds., Hamiltonian Dynamical Systems, a reprint selection (Hilger, Bristol, 1987).

[3] J.E. Howard and S.M. Hohs, Phys. Rev. A 29 (1984) 418.

[4] C. Baesens, J. Guckenheimer, S. Kim and R.S. MacKay, Three coupled oscillators: modelocking, global bifurcations and toroidal chaos, Univ. of Warwick preprint (1990).

[5] J.P. van der Weele, H.W. Capel, T.P. Valkering and T. Post, Physica A 147 (1988) 499.

[6] R.S. MacKay, Introduction of the dynamics of area-preserving maps, in: Proc. US Particle Accelerator School, M. Month, ed. (SLAC, Stanford, 1985).

[7] T.P. Valkering and S.A. van Gils, Bifurcation of periodic orhits near a frequency maximum in near-integrable driven oscillators with friction, Univ. of Twente preprint (June 1990).

[8] T. Poston and I. Stewart, Catastrophe Theory and its Applications (Pitman, London, 1978).

[9] A.A. Chernikov, R.Z. Sagdeev and G.M. Zaslavsky, Physica D 33 (1988) 65.

[10] A. Gerasimov, F.M. Israilev, J.L. Tennyson and A.B. Temnykh, Springer Lecture Notes in Physics, vol. 247 (Springer, Berlin, 1986) p. 154; reprinted in: R.S. MacKay and J.D. Meiss. eds., Hamiltonian Dynamical Systems, a reprint selection (Hilger, Bristol, 1987).

[11] R. Moeckel, Generic bifurcations of the twist coefficient, ETH preprint, Zürich (1988). 